\title{
Síndrome de Lemierre. Caso y revisión de la literatura
}

\author{
Pedro Grille*, Lucciano Grasiuso ${ }^{\dagger}$, Henry Albornoz ${ }^{\ddagger}$
}

\section{Resumen}

El síndrome de Lemierre constituye una entidad poco frecuente y potencialmente grave que puede complicar una infección orofaríngea. Incluye una tromboflebitis de la vena yugular interna y embolias sépticas a distancia. Presentamos el caso clínico de un hombre joven que se presentó, luego de una infección respiratoria alta, con una trombosis de vena yugular interna, seno sigmoideo y transverso izquierdos, asociando además tromboembolismo pulmonar séptico y empiema pleural. Mostró buena evolución con antibioticoterapia empírica, drenaje pleural y anticoagulación. Realizamos una revisión de la literatura sobre este tema enfatizando los aspectos clínicos, epidemiológicos y microbiológicos.

Palabras clave: Síndrome de Lemierre Tromboflebitis

Tromboembolia Embolia pulmonar

Key words: Lemierre syndrome Thrombophlebitis Thromboembolism Pulmonary embolism

\section{Introducción}

El síndrome de Lemierre es una complicación rara y grave de una infección orofaríngea que resulta en la tromboflebitis de la vena yugular interna y embolias sépticas a distancia, generalmente producida por Fusobacterium necrophorum. Su incidencia parece haber aumentado en los últimos años. Esto, sumado a la poca evidencia y diferencias en sus criterios diagnósticos, configura una entidad de singular interés clínico y microbiológico. Presentamos el caso clínico seguido de una revisión de la bibliografía sobre este tema.

\section{Caso clínico}

Hombre de 43 años, con antecedentes personales de tabaquista intenso, bronquítico crónico, alcoholista intenso, consumidor de marihuana y cocaína. Consulta en centro asistencial de segundo nivel por cuadro de cuatro días dado por fiebre, tos, expectoración y dolor facial. Con diagnóstico presuntivo de sinusitis aguda se inicia tratamiento ambulatorio con amoxicilina-clavulánico. Evoluciona con aumento de tos y expectoración, persistencia de fiebre (hasta $39^{\circ} \mathrm{C}$ ) y disnea. Reconsulta e ingresa a sala hospitalaria. Radiografía de tórax evidencia derrame pleural izquierdo, realizándose toracocentesis diagnóstica que confirma empiema de acuerdo con criterios de Light. También se observan múltiples opacidades parenquimatosas periféricas con cavitaciones centrales, compatibles con embolias sépticas pulmonares (figura 1). Agrega insuficiencia respiratoria tipo I y disfunción hemodinámica, por lo que ingresa a la unidad de cuidados intensivos (UCI). De la analítica se

\footnotetext{
* Médico intensivista coordinador, UCI Hospital Maciel, ASSE, Montevideo, Uruguay. Ex Prof. Adj. de la Cátedra de Medicina Intensiva, Facultad de Medicina, Universidad de la República.

† Médico residente de Medicina Intensiva. UCI Hospital Maciel, ASSE, Montevideo, Uruguay.

‡ Médico intensivista de guardia, UCI Hospital Maciel, ASSE, Montevideo, Uruguay. Prof. Adj. de la Cátedra de Enfermedades Infecciosas, Facultad de Medicina, Universidad de la República.

Unidad de Cuidados Intensivos del Hospital Maciel, Administración de los Servicios de Salud del Estado (ASSE), Montevideo, Uruguay.

Correspondencia: Dr. Pedro Grille. Correo electrónico: grillepm@gmail.com

Declaramos no tener conflicto de interés ni recibir financiación.

Cuenta con consentimiento informado del paciente.

Recibido: $31 / 1 / 20$

Aprobado: $2 / 6 / 20$
} 


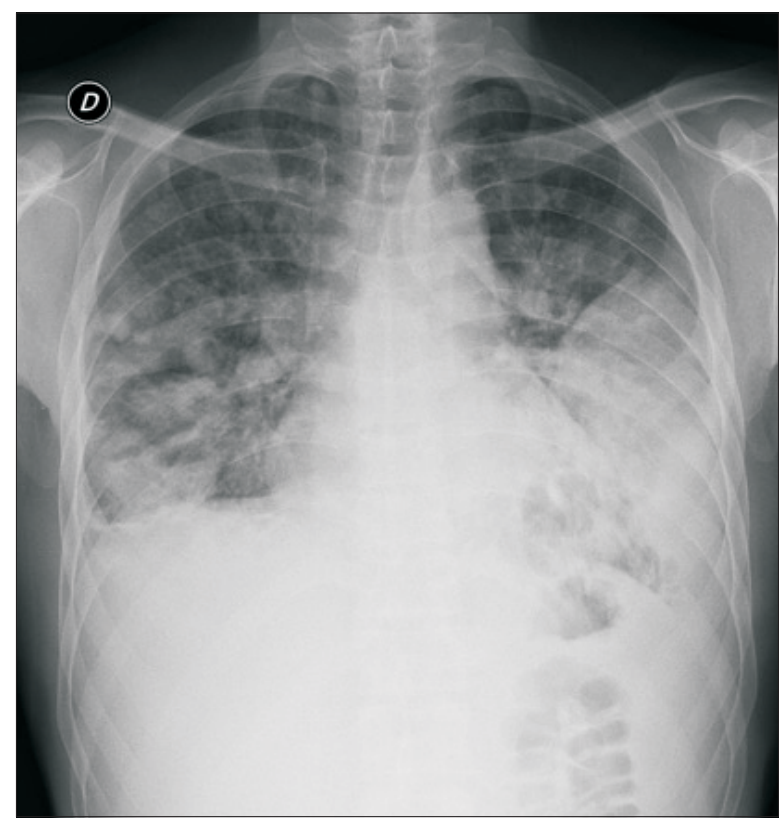

Figura 1. Radiografía de tórax inicial. Se observa derrame pleural izquierdo y varias opacidades parenquimatosas periféricas.

destaca: leucocitosis $21.700 / \mathrm{mm}^{3}$, hemoglobina 7,7 $\mathrm{g} / \mathrm{dl}$, plaquetas $514.000 / \mathrm{mm}^{3}$, tasa de protrombina $67 \%$, fibrinogenemia $629 \mathrm{mg} / \mathrm{dl}$. Antígeno neumocócico en orina, estudio de secreciones respiratorias con coloración de Zehil-Neelsen y serología para VIH: negativos. Hemocultivos, cultivos de secreciones respiratorias y cultivos de líquido pleural sin desarrollo bacteriano. Se realiza angiotomografía computada que evidencia: a) en tórax: derrame pleural izquierdo de moderada entidad, fina lámina de derrame pleural derecho y pericárdico, múltiples consolidaciones parenquimatosas de distribución periférica y parcheada con áreas de cavitación en su interior; b) en cabeza y cuello: trombosis de la vena yugular izquierda que compromete afluente facial a nivel de cuello con extensión a nivel cefálico hasta seno sigmoideo y transverso izquierdos, ocupación parcial del oído medio izquierdo con ocupación total de celdillas mastoideas, y c) abdomen: sin alteraciones (figuras 2, 3 y 4). Ecocardiograma que no evidencia vegetaciones. Se inició tratamiento con reposición hidroelectrolítica, oxigenoterapia, vasopresores, antibioticoterapia (planes sucesivos: piperacilina-tazobactam, ceftazidime-amikacina y luego meropenem), y anticoagulación con heparina de bajo peso molecular. Se realizó drenaje quirúrgico pleural con toracostomía mínima bilateral, hallándose en lóbulo pulmonar inferior izquierdo dos áreas confluentes abscedadas con drenaje espontáneo de pus grisáceo. Permaneció 22 días en UCI, presentando buena evolución clínica.

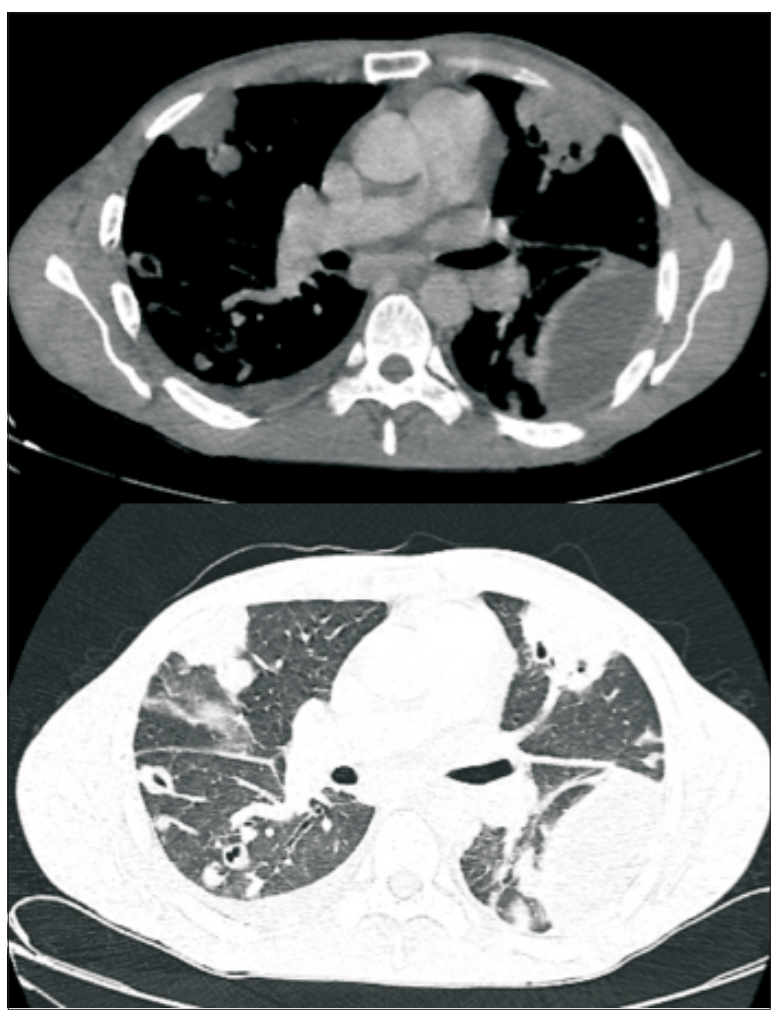

Figura 2. Tomografía computada de tórax. Se observa derrame pleural izquierdo loculado y múltiples opacidades parenquimatosas periféricas con cavitaciones centrales.

\section{Discusión}

La presentación de tromboembolismo pulmonar séptico, asociado a trombosis de vena yugular izquierda en un paciente que cursó una infección respiratoria alta previamente, nos hace plantear el diagnóstico de síndrome de Lemierre.

Dicho síndrome, descrito por primera vez en 1936 por André Lemierre, ha sido aplicado en forma ampliada e indistinta a casos clínicos que incluyan alguno de los siguientes elementos: faringitis aguda complicada con tromboflebitis séptica, embolia séptica a distancia o cultivo positivo para Fusobacterium necrophrum ${ }^{(1)}$.Desde su descripción original, se han desarrollado diferentes definiciones y criterios diagnósticos que básicamente se centran en una complicación secundaria a infección orofaríngea aguda que se caracteriza por una tromboflebitis séptica de la vena yugular interna (84\%) e infecciones metastásicas (100\%), siendo la embolia pulmonar la más frecuente ${ }^{(2)}$. Puede presentarse como sepsis ("sepsis posangina") en $50 \%$, en tanto que la afectación del sistema nervioso central (SNC) se da en $4 \%^{(3)}$. En base a un análisis meticuloso de 393 casos, Riordan y colaboradores arriban a una definición clínico-microbiológica que, 


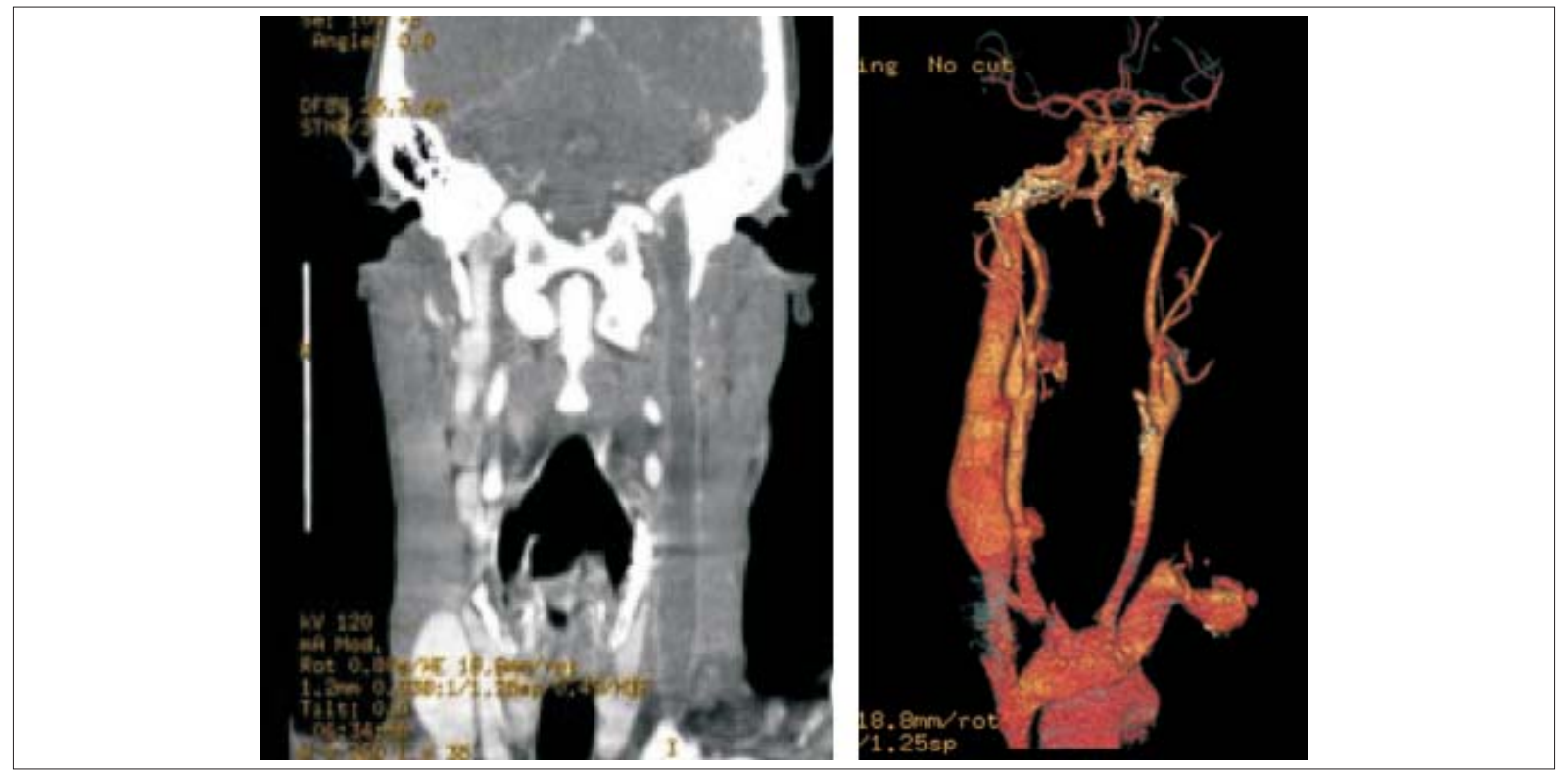

Figura 3. Angiotomografía de vasos de cuello. Se observa defecto de relleno en toda la extensión de la vena yugular interna izquierda.

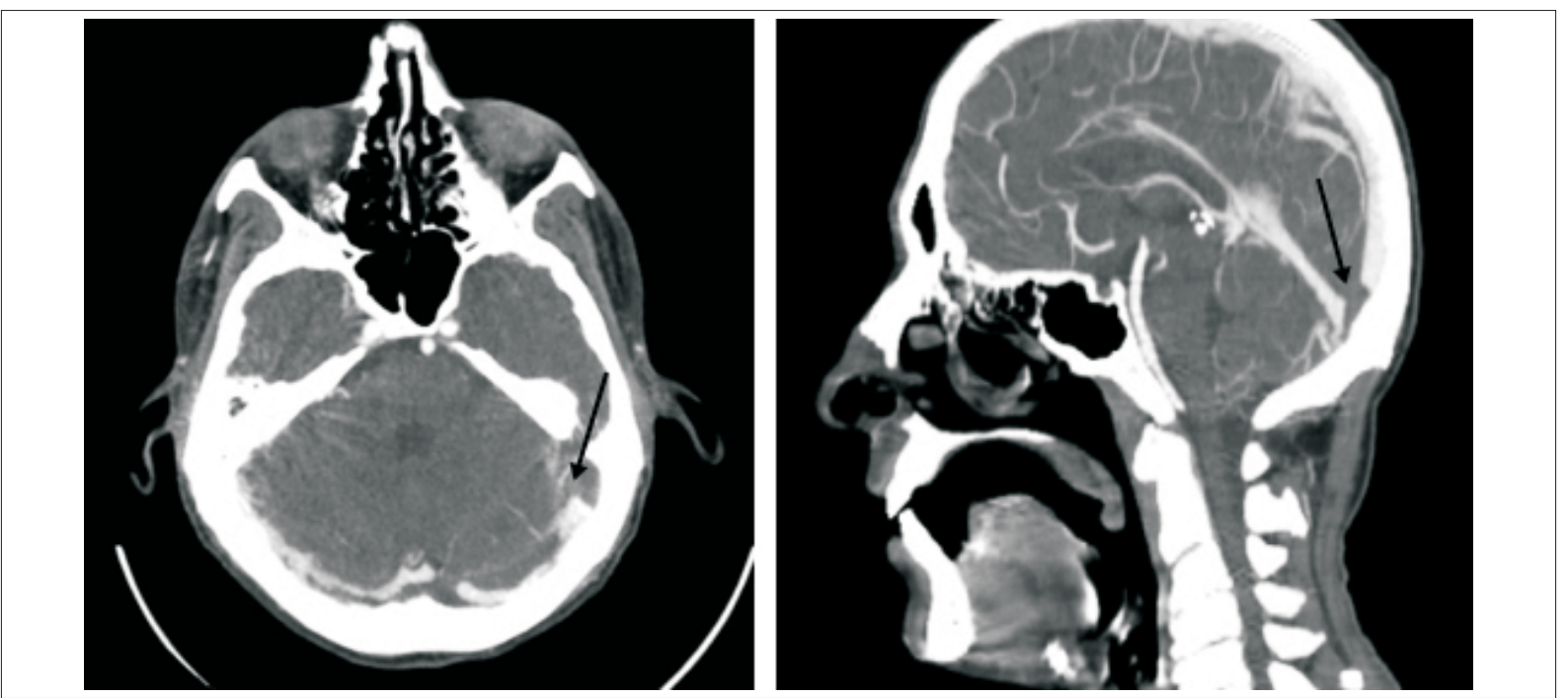

Figura 4. Angiotomografía de vasos intracraneanos. Se visualiza defecto de relleno parcial del seno transverso y sigmoide izquierdos.

si bien no coincide con la definición original de Lemierre, reúne criterios diagnósticos más modernos ${ }^{(4)}$. La misma debe incluir todos los siguientes elementos: 1) faringitis en las cuatro semanas previas a sospecha o infección compatible de región de cuello, oídos o senos faciales; 2) tromboflebitis de la yugular interna o aislamiento de Fusobacterium en hemocultivos o sitios estériles; 3) embolias sépticas a pulmón u otros sitios.
Los datos epidemiológicos de esta entidad presentan cierto grado de confusión debido a su rareza, diferentes definiciones y múltiples aislamientos etiológicos. Más allá de esto, es evidente que fue frecuente en la era preantibiótica, para luego casi desaparecer. En los últimos años, diferentes autores han advertido un aumento en el número de casos, lo que podría explicarse por varios factores: restricción en la prescripción de antibióticos para el tratamiento de la faringitis, aumento de utili- 
zación de macrólidos (no activos frente a F. Necrophorum) y disminución de indicaciones de tonsilectomías ${ }^{(5)}$. De acuerdo a una búsqueda bibliográfica en la base de datos PubMed, de trabajos en idioma español e inglés y utilizando el término "síndrome de Lemierre" o "Lemierre's syndrome" como palabra clave, se encontraron 225 reportes de casos desde 1982 a 2019, siendo la mayoría descripciones de casos clínicos y estudios retrospectivos. Predomina en personas jóvenes (media de edad de 22 años, con el $51 \%$ de los casos en la segunda década de vida), previamente sanas, y con una incidencia variable dependiendo de distintas series entre 3,6 a 14 casos por millón de personas, con una relación hombre/mujer $2: 1^{(2,6)}$. Los sitios más frecuentes de origen de infección son, en orden decreciente, amígdalas, faringe, otitis y sinusitis. Las complicaciones embólicas se localizan más frecuentemente en pulmón, que representa el $85 \%$, y cuya forma de manifestación son la formación de abscesos, derrame pleural, empiema y nódulos cavitados. Otros sitios menos frecuentes son: articulaciones grandes, hígado, bazo, riñón, y más raro, el $\mathrm{SNC}^{(2,3,6)}$. En nuestro paciente las embolias fueron hacia el pulmón con formación de nódulos cavitados, presentando además trombosis venosa cerebral como extensión retrógrada de la trombosis venosa yugular interna.

Fusobacterium necrophorum es el microorganismo más comúnmente involucrado en su etiología. Se trata de un bacilo gramnegativo anaerobio obligado, no fermentador, productor de esporas ${ }^{(5,7-9)}$. Es un comensal presente en la orofaringe, tracto genital femenino y tracto digestivo del ser humano, no estando claro cuál es el factor que determina su patogenicidad ${ }^{(7,10,11)}$. F. necrophorum es causante de la "forma típica" de esta entidad o bifásica, descrita originariamente por Lemierre, como una faringitis inocua y la aparición secundariamente de tromboflebitis o embolias sépticas. Otros microorganismos involucrados son: Bacteroides sp, Eikenella, Streptococcus sp, Peptostreptococcus, Porphyromonas, Prevotella, Klebsiella pneumoniae, Proteus sp y Staphylococcus aureus ${ }^{(3,5)}$. En un porcentaje no despreciable (33\% según la revisión de Johannesen y colaboradores), no se encuentran microorganismos en los cultivos, como sucedió en nuestro caso. Esto puede deberse a un falso negativo por antibioticoterapia previa o a las características de anaerobio obligado de $F$. necrophorum, que determina dificultades en su cultivo in vitro ${ }^{(3)}$.

La patogenia no está bien definida. Sin embargo y más allá de las variantes clínicas, pueden reconocerse tres etapas o estadios en su génesis: 1) infección orofaríngea; 2) diseminación a través del espacio parafaríngeo con tromboflebitis de la vena yugular interna; 3) y luego, embolias sépticas a distancia por vía hematógena. Se han descrito algunos factores de virulencia de $F$. ne- crophorumo: hemolisinas que reducen oxígeno favoreciendo un ambiente anaerobio y crecimiento bacteriano; factores neutrofílicos citotóxicos para evadir el sistema inmune; producción de enzimas proteolíticas para avanzar en la invasión; hemoglutininas que actúan por medio de la agregación plaquetaria con la posterior formación del trombo que, a su vez, induce trombocitopenia; leucotoxinas que favorecerían la formación de abscesos y, por último, lipopolisacárido que participaría en la generación de sepsis y shock séptico ${ }^{(6,10)}$.

No existen guías terapéuticas consensuadas. Las recomendaciones están basadas en la experiencia clínica y estudios observacionales o casos clínicos. Las directivas terapéuticas incluyen la terapia antimicrobiana y el drenaje quirúrgico de las colecciones supuradas en los sitios de infección primarios y secundarios (embólicos). No hay un plan antimicrobiano preestablecido o consensuado. F. necrophorum presenta sensibilidad a metronidazol, clindamicina, betalactámicos combinados con inhibidores de betalactamasa, siendo intrínsicamente resistente a fluorquinolonas y aminoglucósidos ${ }^{(5,8,11)}$. Las cepas productoras de betalactamasas de Fusobacterium necrophorum son raras, pero han sido reportadas ${ }^{(7,12)}$. Algunos regímenes que se pueden utilizar incluyen ampicilina/sulbactam, piperacilina/tazobactam, penicilina más metronidazol o clindamicina, y eventualmente carbapenémicos. Riordan enfatiza en la utilización de metronidazol dada su profunda actividad contra todas las cepas de Fusobacterium sp, su buena penetración tisular y su excelente biodisponibilidad vía oral. La duración de la terapia antimicrobiana promedio, según las diferentes series, es de 4 a 6 semanas (varía entre 10 días y 8 semanas), recomendándose el cambio a la vía oral a las dos semanas $^{(3,6,8)}$.

La anticoagulación para limitar eventos tromboembólicos es controvertida, no existiendo estudios controlados en este sentido. Su indicación es variable según las diferentes series ( $21 \%$ a $64 \%$ ), dado el riesgo de diseminar la infección según algunos autores. Algunos elementos son señalados como indicación de anticoagulación, entre los que se destacan: trombosis venosa cerebral, alta carga trombótica definida como trombosis bilateral o retrógrada hacia el SNC, complicaciones embólicas, infarto arterial isquémico, pobre respuesta a antibioticoterapia, infarto cerebral y trombofilia. No hay consenso sobre la duración de la anticoagulación, siendo también variable entre las diferentes series (de 5 a 12 semanas) $)^{(6,7,11,13,14)}$.

El tratamiento quirúrgico, entendido como drenaje de abscesos, colecciones y debridamiento de tejido necrótico, es un pilar fundamental en el manejo del síndrome de Lemierre ${ }^{(6)}$. Tanto la ligadura como la resección de la vena trombosada era una práctica habitual en la era 
preantibiótica. Hoy día no se utiliza o se reserva para los casos de sepsis no controlada con tratamiento convencional o persistencia de embolias sépticas a pesar de un adecuado tratamiento antibiótico ${ }^{(6,7,10-12,15-17)}$.

El pronóstico de esta entidad ha cambiado drásticamente con el tratamiento antimicrobiano. Su mortalidad ha disminuido desde casi $90 \%$ a menos de $5 \%$ en la actualidad $^{(3,5)}$

\section{Summary}

Lemierre's syndrome constitutes a rare and potentially serious entity, that may complicate oropharyngeal infections. This condition includes thrombophlebitis of the internal jugular vein and distant septic embolisms. The study presents the clinical case of a young man who consulted after an upper respiratory infection, with internal jugular vein thrombosis, left sigmoid and transverse seins, also associating septic pulmonary embolism and pleural empyema. The patient had a positive evolution after empirical antibiotic, pleural drainage and anticoagulants. We conducted literature review on this condition, focusing on clinical, epidemiological and microbiological aspects.

\section{Resumo}

A síndrome de Lemierre é uma doença rara e potencialmente grave que pode complicar uma infecção orofaríngea. Inclui uma tromboflebite da veia jugular interna e embolias sépticas a distância. Descrevemos o caso clínico de um homem jovem que depois de uma infecção respiratória alta apresentou trombose de veia jugular interna, seio sigmoide e transverso esquerdos, associado com tromboembolismo pulmonar séptico e empiema pleural. Teve boa evolução com antibioticoterapia empírica, drenagem pleural e anticoagulação. Realizamos uma revisão da literatura sobre este tema enfatizando os aspectos clínicos, epidemiológicos e microbiológicos.

\section{Bibliografía}

1. Lemierre A. On certain septicaemia due to anaerobic organisms. Lancet 1936; 227(5874):701-3.

2. Rivero Marcotegui M, Sánchez Rodríguez C, Cabal Soto S, Aizcorbe Garralda M. Síndrome de Lemierre y mycoplasma pneumoniae. An Med Interna (Madrid) 2005; 22(11):541-3.
3. Johannesen KM, Bodtger U. Lemierre's Syndrome: current perspectives on diagnosis and management. Infect Drug Resist 2016; 9:221-7.

4. Riordan T, Kuppalli K, Livorsi D, Talati NJ, Osborn M. Lemierre's Syndrome due to fusobacterium necrophorum. Lancet Infect Dis 2007; 20:622-59.

5. Karkos PD, Asrani S, Karkos CD, Leong SC, Theochari EG, Alexopoulou TD, et al. Lemierre's Syndrome: a systematic review. Laryngoscope 2009; 119(8):1552-9.

6. Kuppalli K, Livorsi D, Talati NJ, Naasha J, Osborn M. Lemierre's Syndrome due to fusobacterium necrophorum. Lancet Infect Dis 2012; 12(10):808-15.

7. Kristensen LH, Prag J. Human necrobacillosis, with emphasis on Lemierre's Syndrome. Clin Infect Dis 2000; 31(2):524-32.

8. Riordan T. Human infection with fusobacterium necrophorum (Necrobacillosis), with a focus on Lemierre's Syndrome. Clin Microbiol Rev 2007; 20(4):622-59.

9. Tellería Martín A, Jiménez Urra I, Susperregui Insausti I, Ramos Castro J, Gutierrez Oliver YA. Sindrome de Lemierre. Med Intensiva 2005; 29(8):441-4.

10. Montiel Crespo R, Quintero Otero S, Hernandez Gonzalez A, de Benito Guerra MT, García Trujillo I, Tinoco Rasero I, et al. Sindrome de Lemierre: la enfermedad olvidada. Med Intensiva 2005; 29(8):437-40.

11. Alferijat M. A case of Lemierre's Syndrome with a brief literature review. J Infect Public Health 2016; 9:681-3.

12. Leoncini E, Ricciardi W, Cadoni G, Arzani D, Petrelli L, Paludetti G, et al. Adult height and head and neck cancer: a pooled analysis within the INHANCE Consortium. Head Neck 2014; 36(10):1391.

13. Golpe R, Marín B, Alonso M. Lemierre 's Syndrome (Necrobacillosis). Postgrad Med J 1999; 75(881):141-4.

14. Eilbert W, Singla N. Lemierre 's syndrome. Int J Emerg Med 2013; 6(1):40.

15. Rebelo J, Nayan S, Choong K, Fulford M, Chan A, Sommer DD. To anticoagulate? Controversy in the management of thrombotic complications of head \& neck infections. Int J Pediatr Otorhinolaryngol 2016; 88:129-35.

16. De Smet K, Claus PE, Alliet G, Simpelaere A, Desmet G. Lemierre's syndrome: a case study with a short review of literature. Acta Clin Belg 2019; 74(3):206-10.

17. Cantero Macedo AM, Carrasco Seco MC, Forte Guerrero M, Bazo Fariñas AV, Callejo Calvo J. Lemierre's syndrome, a serious complication of acute sinusitis. Actual Med 2016; 101(798):112-7.

\section{Contribución de autores}

Los autores han participado en las siguientes etapas del manuscrito:

Pedro Grille, https://orcid.org/0000-0002-2099-4378. Concepción, diseño, ejecución, análisis, redacción y revisión crítica.

Lucciano Grasiuso, https://orcid.org/0000-0001-7559-6193. Ejecución, análisis y redacción.

Henry Albornoz, https://orcid.org/0000-0003-4491-830X. Análisis y revisión crítica. 